\title{
PO3-6-3
}

Poster session

\section{Visualization of advanced glycation end-products phagocytosis by macrophages and identification of possible receptor}

\author{
Shinichi Hamasaki ${ }^{1}$, Takuro Kobori ${ }^{2}$, Atsuhiro Kitaura ${ }^{1}$, Yui Yamazaki ${ }^{2}$, Takashi Nishinaka ${ }^{2}$, \\ Atsuko Niwa ${ }^{2}$, Shinichi Nakao ${ }^{1}$, Masahiro Nishibori ${ }^{3}$, Hideo Takahashi ${ }^{2}$ \\ ${ }^{1}$ Department of Anesthesiology, Kindai University, Faculty of Medicine, Japan, ${ }^{2}$ Department of Pharmacology, Kindai \\ University, Faculty of Medicine, Japan, ${ }^{3}$ Department of Pharmacology, Okayama University, Graduate School of \\ Medicine, Dentistry and Pharmaceutical Sciences, Japan
}

Background Advanced glycation end-products (AGEs), which are formed by nonenzymatic glycation reaction of reducing sugars and proteins, accumulates in the body due to the persistence of hyperglycemia, resulting in higher values in diabetic patients. Macrophage is capable of eliminating AGEs possibly via its phagocytic activity, and is therefore involved in the progression of arteriosclerosis. However, it has yet to be determined how macrophages uptake AGEs. The aim of this study is to visualize the process of AGEs phagocytosis in macrophages and to identify the surface scavenger receptor involved in AGEs uptake.

Methods RAW264.7 cells, the mouse macrophage-like cell line, were incubated with glyceraldehyde-derived AGE (AGE -2) and glycolaldehyde-derived AGE (AGE-3) at different concentrations ranging from $0.2-200 \mu \mathrm{g} / \mathrm{mL}$ for 4 hrs. The uptake level of AGEs and the expression levels of surface scavenger receptors in RAW264.7 cells were analyzed by flow cytometry. The intracellular localization of AGEs phagocytosed by RAW264.7 cell and its morphological changes were visualized by immunofluorescence microscopy and immunoelectron microscopy.

Results The uptake level of AGEs $200 \mu \mathrm{g} / \mathrm{mL}$ was increased significantly from $10 \mathrm{~min}$ to $4 \mathrm{hrs}$, while there were little changes in that of AGEs used at $0.2 \mu \mathrm{g} / \mathrm{mL}$. By means of immunofluorescence microscopy and immunoelectron microscopy, AGEs were found to be localized in the intracellular phagosome of RAW264.7 cells. Of particular interest, RAW264.7 cells extended their pseudopodia or end-feet toward AGEs, along with the morphological alteration like an elongated fibroblast. Furthermore, AGEs treatment significantly enhanced the surface expression levels of several scavenger receptors including CD204 in RAW 264.7 cells. Interestingly, neutralizing antibody against CD204 but not against others dramatically inhibited the uptake levels of AGEs.

Conclusions We discovered here that AGEs treatment induces the morphological alteration in macrophages to phagocytose AGEs themselves. Furthermore, CD204 may be responsible for mediating AGEs uptake in macrophages. 